\title{
More on FOX News: FOXA1 on the horizon of estrogen receptor function and endocrine response
}

\author{
Xiaoyong Fu' ${ }^{1,2,3,4}$, Catherine Huang ${ }^{1,2,3,4}$ and Rachel Schiff*1,2,3,4
}

\begin{abstract}
Estrogen receptor a (ER) is a major driver of breast cancer and the target of endocrine therapy. Full disclosure of the cofactors regulating ER interactions with chromatin and its transcriptional regulatory activity is still elusive. Novel genome-wide profiling tools have mapped ER binding events in breast cancer cells and delineated cofactors important in ER activity. Among these, the Forkhead protein FOXA1 is emerging as a key factor dictating global chromatin structure and the transcriptional function of ER in breast and non-breast cancer cells. The significance of FOXA1 in the chromatin interactions and transcriptional regulation of both estrogen- and tamoxifen-bound $E R$, and in supporting tamoxifen-resistant cell growth, may impact current endocrine therapies.
\end{abstract}

\section{Background}

The estrogen receptor $\alpha(E R)$ protein is present in over two-thirds of breast cancers, where it functions in the nucleus as a ligand-dependent transcription factor to drive cell proliferation, survival, and invasiveness. Endocrine therapies to block ER activity are the most important systemic treatments for ER-positive breast cancers, though resistance is prevalent [1]. We need to understand the molecular determinants regulating ER DNA binding and activity to elucidate the mechanisms underlying this resistance.

The advancement of chromatin immunoprecipitation (ChIP)-based technologies, which combine ChIP with microarrays or high throughput sequencing (ChIP-seq), has helped to identify a network of co-regulators and

*Correspondence: rschiff@bcm.edu

'Lester and Sue Smith Breast Center, Baylor College of Medicine, One Baylor Plaza, BCM600, Houston, TX 77030, USA

Full list of author information is available at the end of the article their genome-wide DNA binding sites (known as their cistrome) that cooperate to regulate ER DNA binding and transcriptional activity. These technologies have revealed that, in breast cancer cells, ER mostly binds to distal enhancers that are also enriched for Forkhead motifs [2-4]. Furthermore, the Forkhead protein FOXA1, a favorable prognostic factor that correlates with the luminal A breast cancer subtype and hormonal sensitivity [5], has been shown to act as a pioneer factor, opening chromatin regions for the recruitment of ER to these DNA binding sites [6]. However, how global the importance of FOXA1 is in mediating ER function in breast cancer, as well as in other target tissues and under different ligand conditions, and what are the underlying factors that determine FOXA1 specificity remain open questions.

\section{The article}

To more broadly investigate the genome-wide relationship of ER and FOXA1 DNA-binding sites, Hurtado and colleagues [7] first performed ChIP-seq of ER and FOXA1 in three different breast cancer cell lines. FOXA1 binding events were found to be dynamic and cell-linespecific, a phenomenon potentially related to the insulator protein CTCF. Within each cell line, a significant overlap of over 50\% was found between ER and FOXA1 sites. FOXA1 was also found to mediate ER function in non-breast cancer cells and to act upstream of ERchromatin interactions, enabling ER binding at more condensed chromatin regions. Additionally, FOXA1 was required to globally facilitate ER-mediated transcription, since downregulation of FOXA1 affected the transcription of more than 95\% of estrogen-regulated genes. Finally, FOXA1 knockdown resulted in significant growth inhibition of MCF7 cells, substantiating the key role of FOXA1 in the estrogen response of breast cancer cells.

To study the ER cistromic profile and the role of FOXA1 in mediating tamoxifen inhibition, estrogendeprived MCF-7 cells treated with estrogen or tamoxifen were subjected to ER ChIP-seq and gene expression microarray analyses. In contrast to a previous report [8], 
the results demonstrated that tamoxifen induced ER binding events similar to those induced by estrogen. Additionally, estrogen and tamoxifen were found to regulate common genes. FOXA1 knockdown showed that tamoxifen-ER employs similar FOXA1-dependent mechanisms as estrogen to interact with chromatin. However, the experimental setting prevented direct assessment of whether FOXA1 is required for the tamoxifen antiproliferative effects in breast cancer cells. Of note, in tamoxifen-resistant derivatives of MCF-7 cells, chromatin binding profiles of both ER and FOXA1 dramatically differ from those of the wild-type cell line, and the binding occurred independently of tamoxifen treatment. However, ER and FOXA1 binding regions still significantly overlapped and, most importantly, ER chromatin binding and cell proliferation in the tamoxifenresistant line still required FOXA1.

\section{The viewpoint}

Unbiased, genome-wide mapping and profiling of ER interaction with chromatin and its transcriptional regulation activity in breast cancer have recently been established by leading groups in this field $[2-4,9,10]$ and have created a valuable resource to increase our basic understanding of estrogen/ER action and to improve therapeutic strategies. These studies have collectively demonstrated the fundamental role of FOXA1 in guiding and regulating ER chromatin binding events and gene transcription. While the recent report of Hurtado and colleagues [7] strongly substantiates these previous data and notions, its novel insights into the role of FOXA1 in both tamoxifen action and resistance, and into mechanistic aspects of FOXA1 action, are of clinical and biological significance.

The pivotal role of FOXA1 in ER-DNA interaction and transcriptional activity, as well as in the growth of wild type MCF7 cells, points to FOXA1 as a potential therapeutic target for opposing ER activity and tumor growth, either alone or in combination with additional endocrine targets. Conversely, since the tamoxifen-ER interaction with DNA, which is a key component of tamoxifen's inhibitory action on ER-dependent gene expression, was also found to depend tightly on FOXA1, one could argue that co-targeting of FOXA1 might antagonize, instead of enhance, the inhibitory capabilities of anti-estrogens such as tamoxifen. Notably, however, in tamoxifen-resistant cells, the extensively altered pattern of ER binding and resistant cell growth phenotype both required FOXA1, an observation pointing again to FOXA1 as a therapeutic candidate. Interestingly, Lupien and colleagues [11] recently showed that epidermal growth factor stimulation of MCF7 cells induces a ligand-independent distinct profile of ER binding sites that is still enriched for Forkhead binding sites. This growth factor (GF)-specific
ER cistrome dictates a unique transcriptional program that correlates with gene expression signatures found in poor-outcome as well as HER2-positive breast cancers [11]. Similarly, a unique ER cistrome and ER transcriptional activation were also documented upon AKT activation [12], and others have shown that blocking this pathway with a PI3K inhibitor can restore the 'classic' pattern of E2-induced gene expression [13]. Acquired tamoxifen resistance of MCF7 cells and xenografts is also associated with increased GF/HER1/2 signaling and a distinct expression profile that correlates with growth factor gene expression signatures [14,15]. As aberrant expression/activation of GF pathways is commonly associated with endocrine resistance [1,15], the data cumulatively imply that the differential ER chromatin binding and subsequently altered transcriptional program that derive from hyperactive GF signaling are fundamental underlying mechanisms of endocrine resistance. The relative contributions to this global molecular switch of ER-chromatin interactions and gene expression provided by (1) non-genomic ER function, (2) additional Forkhead family members, (3) other chromatin remodeling factors and/or ER coregulators, (4) posttranslational modification of these key factors, and (5) additional epigenetic modifications are still open questions. However, the critical roles of FOXA1 and ER in this process, as shown by Hurtado and colleagues [7], indeed suggest that co-targeting of FOXA1 together with more potent ER inhibitors or degraders [11] might represent an improved strategy to circumvent endocrine resistance in breast cancer.

Abbreviations

ChIP, chromatin immunoprecipitation; ER, estrogen receptor a; GF, growth factor.

\section{Competing interests}

The authors declare that they have no competing interests.

\section{Acknowledgements}

This work was supported in part by NCI grants P50 CA58183 (Breast Cancer SPORE) and the Stand Up to Cancer Breast Cancer Program.

\section{Author details}

'Lester and Sue Smith Breast Center, Baylor College of Medicine, One Baylor Plaza, BCM600, Houston, TX 77030, USA. ${ }^{2}$ Dan L Duncan Cancer Center, Baylor College of Medicine, Houston, TX 77030, USA. ${ }^{3}$ Margaret M and Albert B Alkek Department of Medicine, Baylor College of Medicine, Houston, TX 77030, USA. ${ }^{4}$ Department of Molecular and Cellular Biology, Baylor College of Medicine, Houston, TX 77030, USA.

Published: 20 April 2011

\section{References}

1. Osborne CK, Schiff R: Mechanisms of endocrine resistance in breast cancer. Annu Rev Med 2011, 62:233-247.

2. Carroll JS, Meyer CA, Song J, Li W, Geistlinger TR, Eeckhoute J, Brodsky AS, Keeton EK, Fertuck KC, Hall GF, Wang Q, Bekiranov S, Sementchenko V, Fox EA, Silver PA, Gingeras TR, Liu XS, Brown M: Genome-wide analysis of estrogen receptor binding sites. Nat Genet 2006, 38:1289-1297.

3. Joseph R, Orlov YL, Huss M, Sun W, Kong SL, Ukil L, Pan YF, Li G, Lim M, Thomsen JS, Ruan Y, Clarke ND, Prabhakar S, Cheung E, Liu ET: Integrative 
model of genomic factors for determining binding site selection by estrogen receptor-alpha. Mol Syst Biol 2010, 6:456.

4. Laganiere J, Deblois G, Lefebvre C, Bataille AR, Robert F, Giguere V: Location analysis of estrogen receptor alpha target promoters reveals that FOXA1 defines a domain of the estrogen response. Proc Natl Acad Sci U S A 2005, 102:11651-11656.

5. Badve S, Turbin D, Thorat MA, Morimiya A, Nielsen TO, Perou CM, Dunn S, Huntsman DG, Nakshatri H: FOXA1 expression in breast cancer-correlation with luminal subtype A and survival. Clin Cancer Res 2007, 13:4415-4421.

6. Cirillo LA, Lin FR, Cuesta I, Friedman D, Jarnik M, Zaret KS: Opening of compacted chromatin by early developmental transcription factors HNF3 (FoxA) and GATA-4. Mol Cell 2002, 9:279-289.

7. Hurtado A, Holmes KA, Ross-Innes CS, Schmidt D, Carroll JS: FOXA1 is a key determinant of estrogen receptor function and endocrine response. Nat Genet 2010, 43:27-33.

8. Welboren WJ, van Driel MA, Janssen-Megens EM, van Heeringen SJ, Sweep FC, Span PN, Stunnenberg HG: ChIP-Seq of ERalpha and RNA polymerase II defines genes differentially responding to ligands. EMBO J 2009, 28:1418-1428.

9. Gu F, Hsu HK, Hsu PY, Wu J, Ma Y, Parvin J, Huang TH, Jin VX: Inference of hierarchical regulatory network of estrogen-dependent breast cancer through ChIP-based data. BMC Syst Biol 2010, 4:170.

10. Lupien M, Eeckhoute J, Meyer CA, Krum SA, Rhodes DR, Liu XS, Brown M: Coactivator function defines the active estrogen receptor alpha cistrome. Mol Cell Biol 2009, 29:3413-3423.

11. Lupien M, Meyer CA, Bailey ST, Eeckhoute J, Cook J, Westerling T, Zhang X, Carroll JS, Rhodes DR, Liu XS, Brown M: Growth factor stimulation induces a distinct $E R(a l p h a)$ cistrome underlying breast cancer endocrine resistance. Genes Dev 2010, 24:2219-2227.

12. Bhat-Nakshatri P, Wang G, Appaiah H, Luktuke N, Carroll JS, Geistlinger TR, Brown M, Badve S, Liu Y, Nakshatri H: AKT alters genome-wide estrogen receptor alpha binding and impacts estrogen signaling in breast cancer. Mol Cell Biol 2008, 28:7487-7503.

13. Creighton CJ, Fu X, Hennessy BT, Casa AJ, Zhang Y, Gonzalez-Angulo AM, Lluch A, Gray JW, Brown PH, Hilsenbeck SG, Osborne CK, Mills GB, Lee AV, Schiff R: Proteomic and transcriptomic profiling reveals a link between the $\mathrm{PI} 3 \mathrm{~K}$ pathway and lower estrogen-receptor (ER) levels and activity in ER+ breast cancer. Breast Cancer Res 2010, 12:R40

14. Massarweh S, Osborne CK, Creighton CJ, Qin L, Tsimelzon A, Huang S, Weiss $H$, Rimawi M, Schiff $R$ : Tamoxifen resistance in breast tumors is driven by growth factor receptor signaling with repression of classic estrogen receptor genomic function. Cancer Res 2008, 68:826-833.

15. Nicholson Rl, Hutcheson IR, Hiscox SE, Knowlden JM, Giles M, Barrow D, Gee $J \mathrm{M}$ : Growth factor signalling and resistance to selective oestrogen receptor modulators and pure anti-oestrogens: the use of anti-growth factor therapies to treat or delay endocrine resistance in breast cancer. Endocr Relat Cancer 2005, 12 Suppl 1:S29-36.

doi:10.1186/bcr2849

Cite this article as: Fu X, et al: More on FOX News: FOXA1 on the horizon of estrogen receptor function and endocrine response. Breast Cancer Research 2011, 13:307 